\title{
Optical Solitary Waves in the Higher Order Nonlinear Schrödinger Equation
}

\author{
M. Gedalin, T.C. Scott, and Y.B. Band \\ Departments of Chemistry and Physics, Ben-Gurion University of the Negev, \\ 84105 Beer-Sheva, Israel
}

\begin{abstract}
We study solitary wave solutions of the higher order nonlinear Schrödinger equation for the propagation of short light pulses in an optical fiber. Using a scaling transformation we reduce the equation to a two-parameter canonical form. Solitary wave (1-soliton) solutions always exist provided easily met inequality constraints on the parameters in the equation are satisfied. Conditions for the existence of $N$-soliton solutions $(N \geq 2)$ are determined; when these conditions are met the equation becomes the modified KdV equation. A proper subset of these conditions meet the Painlevé plausibility conditions for integrability.
\end{abstract}

42.81.Dp, 02.30.Jr, 42.65.Tg, 42.79.Sz

The propagation of nonlinear waves in dispersive media is of great interest since nonlinear dispersive systems are ubiquitous in nature. Propagation of ultrashort light pulses in optical fibers is of particular interest because of the common expectation that solitary waves may be of extensive use in telecommunication and even revolutionarize it. The existence of solitary wave solutions implies perfect balance between nonlinearity and dispersion which usually requires rather specific conditions and cannot be established in general. The objective of the present paper is to study the conditions under which the existence of solitary waves is guaranteed for ultrashort pulses.

The propagation of light pulses in fibers is well described by the higher order nonlinear Schrödinger equation (HONSE) 佂, a partial differential equation (PDE) whose right hand side includes the effects of group velocity dispersion, self-phase modulation, third order dispersion, self-steepening, and self-frequency shifting via stimulated Raman scattering, respectively:

$$
E_{z}=i\left(\alpha_{1} E_{t t}+\alpha_{2}|E|^{2} E\right)+\alpha_{3} E_{t t t}+\alpha_{4}\left(|E|^{2} E\right)_{t}+\alpha_{5}\left(|E|^{2}\right)_{t} E .
$$

When the last three terms are omitted this propagation equation for the slowly varying envelope of the electric field, $E$, reduces to the nonlinear Schrödinger equations (NSE), which is integrable (meaning it not only admits $N$-solitary wave solutions, but that the evolution of any initial condition is known in principle) 国 6]. We call these $N$-solitary wave solutions $N$-solitons, and mean by this that the solitary waves scatter elastically and asymptotically preserve their shape upon undergoing collisions, just like true solitons. However, for short duration pulses the last three terms are non-negligible and should be retained. In general, the presence or absence of solitary wave solutions depends on the coefficients $\alpha$ appearing in Eq. (11), and therefore, on the specific nonlinear and dispersive features of the medium. Here, we reduce the HONSE to a two-parameter equation and derive a general solitary wave (1-soliton) solution. We determine conditions when $N$-soliton solutions exist and display the solutions. We also study the Painlevé plausibility conditions for integrability and show that these are only a proper subset of the conditions necessary for $N$-soliton solutions to exist.

We begin by scaling the HONSE, letting $E=b_{1} A, z=b_{2} \zeta$, and $t=b_{3} \tau$. Substituting into the HONSE we obtain

$$
A_{\zeta}=i\left(\left(b_{2} \alpha_{1} / b_{3}^{2}\right) A_{\tau \tau}+\left(b_{1}^{2} b_{2}\right) \alpha_{2}|A|^{2} A\right)+\left(b_{2} \alpha_{3} / b_{3}^{3}\right) A_{\tau \tau \tau}+\left(b_{1}^{2} b_{2} \alpha_{4} / b_{3}\right)\left(|A|^{2} A\right)_{\tau}+\left(b_{1}^{2} b_{2} \alpha_{5} / b_{3}\right)\left(|A|^{2}\right)_{\tau} A .
$$

Choosing $b_{1}=\left(\alpha_{1}^{3} /\left(\alpha_{2} \alpha_{3}^{2}\right)\right)^{1 / 2}, b_{2}=\alpha_{3}^{2} / \alpha_{1}^{3}$, and $b_{3}=\alpha_{3} / \alpha_{1}$, we can set the coefficients of the first, second and third terms on the right hand side of Eq. (2) to unity, so that the HONSE becomes

$$
A_{\zeta}=i\left(A_{\tau \tau}+|A|^{2} A\right)+A_{\tau \tau \tau}+\gamma_{1}\left(|A|^{2} A\right)_{\tau}+\gamma_{2}\left(|A|^{2}\right)_{\tau} A,
$$

where $\gamma_{1}=b_{1}^{2} b_{2} \alpha_{4} / b_{3}=\alpha_{4} \alpha_{1} / \alpha_{2} \alpha_{3}$ and $\gamma_{2}=b_{1}^{2} b_{2} \alpha_{5} / b_{3}=\alpha_{5} \alpha_{1} / \alpha_{2} \alpha_{3}$.

A solution to Eq. (3) of the form $A(\zeta, \tau)=y(\tau+\beta \zeta) \exp [i(\kappa \zeta-\Omega \tau)]$, with $y$ real, exists regardless of the values of the parameters $\gamma_{1}$ and $\gamma_{2}$ as can be easily demonstrated by substituting this form into Eq. (3) and equating the real and imaginary parts of the resulting equation. This procedure yields the following necessary and sufficient condition on $\Omega$, and an equation for $\kappa$ in terms of $\Omega$ :

$$
\begin{aligned}
& \Omega=\frac{3 \gamma_{1}+2 \gamma_{2}-3}{6\left(\gamma_{1}+\gamma_{2}\right)}, \quad(\Omega \neq 1 / 3), \\
& \kappa-\Omega^{3}+\Omega^{2}=\left(\beta+3 \Omega^{2}-2 \Omega\right)(1-3 \Omega) .
\end{aligned}
$$


The function $y(\xi)(\xi=\tau+\beta \zeta)$ satisfies the ordinary differential equation

$$
y_{\xi \xi}=\left(\beta+3 \Omega^{2}-2 \Omega\right) y-\frac{3 \gamma_{1}+2 \gamma_{2}}{3} y^{3},
$$

whose solution is given generally in terms of doubly periodic elliptic functions. For zero energy (i.e., for $\left.y_{\xi}^{2}-\left(\beta+3 \Omega^{2}-2 \Omega\right) y^{2}+\left(3 \gamma_{1}+2 \gamma_{2}\right) y^{4} / 6=0\right)$ we find the solitary wave solution

$$
\begin{aligned}
& A(\zeta, \tau)=\left(\frac{6\left(\beta+3 \Omega^{2}-2 \Omega\right)}{3 \gamma_{1}+2 \gamma_{2}}\right)^{1 / 2} \cosh ^{-1}\left[\left(\beta+3 \Omega^{2}-2 \Omega\right)^{1 / 2}(\tau+\beta \zeta)\right] \\
& \times \exp \left\{i\left[\left(\left(\beta+3 \Omega^{2}-2 \Omega\right)(1-3 \Omega)+\Omega^{3}-\Omega^{2}\right) \zeta-\Omega \tau\right]\right\}
\end{aligned}
$$

provided $\left(\beta+3 \Omega^{2}-2 \Omega\right)>0$ and $3 \gamma_{1}+2 \gamma_{2}>0$. Thus, solitary wave solutions always exist (in contrast with what is implied in Ref. [7]) provided $3 \gamma_{1}+2 \gamma_{2}>0$. For $\Omega=0$ (i.e., for $3 \gamma_{1}+2 \gamma_{2}=3$ ) the solitary wave solution reduces to $A(\zeta, \tau)=2^{1 / 2} \eta \cosh ^{-1}\left(\eta\left(\tau+\eta^{2} \zeta\right)\right) \exp \left(i \eta^{2} \zeta\right)$ where $\kappa=\beta \equiv \eta^{2}>0$.

The case of $\Omega=1 / 3$ is very special, not only because Eq. (1) (which should be written in the form $3 \gamma_{1}+2 \gamma_{2}=$ $\left.3\left(1-\gamma_{1} \Omega\right) /(1-3 \Omega)\right)$ is not applicable, but also because Eq. (3) is expected to be integrable for this case, as we shall show below using a Painlevé analysis [8]10]. The Painlevé condition for integrability (see below)

$$
\gamma_{1}=3, \gamma_{2}=-3 / 2
$$

(which differs from the result claimed in Ref. [7]) yields $\Omega=1 / 3$ and $\kappa=-2 / 27$, and the solitary wave solution takes the form

$$
A(\zeta, \tau)=(\beta-1 / 3)^{1 / 2} \cosh ^{-1}\left[(\beta-1 / 3)^{1 / 2}(\tau+\beta \zeta)\right] \exp [-i(2 \zeta / 27+\tau / 3)] .
$$

It is of interest to compare the solitary wave solutions for different values of parameters $\gamma_{1}$ and $\gamma_{2}$. All solitary waves have intensity profiles of the form $I(\zeta, \tau)=|A(\zeta, \tau)|^{2}=I_{s} \cosh ^{-2}\left[(\tau+\beta \zeta) / \tau_{s}\right]$. The solitary wave width $\tau_{s}$ and its intensity $I_{s}$ are related by the expression $I_{s} \tau_{s}^{2}=6 /\left(3 \gamma_{1}+2 \gamma_{2}\right)$ (where $3 \gamma_{1}+2 \gamma_{2}>0$ for a solitary wave solution). For comparison, recall that $I_{s} \tau_{s}^{2}=2$ for the NSE solitary wave $\left(\alpha_{3}=\alpha_{4}=\alpha_{5}=0\right)$, so that for equal $\tau_{s}$, $I_{s}^{\mathrm{HONSE}} / I_{s}^{\mathrm{NSE}}=3 /\left(3 \gamma_{1}+2 \gamma_{2}\right)$. The width $\tau_{s}$ and $\beta$ (the negative of the inverse-velocity in the coordinate system moving with the group velocity of the light pulse, i.e., the solitary wave velocity $v_{s}=v_{g}-\beta^{-1}$ where $v_{g}$ is the group velocity) are related by the equation $\tau_{s}=\left(\beta+3 \Omega^{2}-2 \Omega\right)^{-1 / 2}$ where $\Omega$ is given by Eq. (4).

As is well-known, existence of solitary wave solutions does not guarantee existence of $N$-soliton solutions with $N>1$. In the absence of the inverse scattering solution for the HONSE, the Hirota method [11], based on the cutoff of the Padé-approximation [12] $A=G / F, F$ being real, is often useful. Direct substitution of this representation into the PDE in Eq. (3) can be expedited using Hirota $D$ operators 11] (defined by its operation on bilinear forms $\left.D_{t}(f \cdot g):=\left.\left(\partial / \partial t-\partial / \partial t^{\prime}\right) f(t) g\left(t^{\prime}\right)\right|_{t=t^{\prime}}\right)$ and by separation of the linear part of the PDE to yield:

$$
\begin{aligned}
& \left(D_{\zeta}-i D_{\tau}^{2}-D_{\tau}^{3}\right)(G \cdot F)=0 \\
& (G \cdot F)\left[-i D_{\tau}^{2}(F \cdot F)+i\left(G^{*} \cdot G\right)+\left(\gamma_{1}+\gamma_{2}\right) D_{\tau}\left(G^{*} \cdot G\right)\right] \\
& +D_{\tau}(G \cdot F)\left[-3 D_{\tau}^{2}(F \cdot F)+\left(3 \gamma_{1}+2 \gamma_{2}\right)\left(G^{*} \cdot G\right)\right]=0 .
\end{aligned}
$$

The standard algorithm is to further substitute a 2-soliton solution in a power series in $\epsilon$ of the form

$$
\begin{aligned}
& G=\epsilon\left(\exp \left(\theta_{1}\right)+\exp \left(\theta_{2}\right)\right)+\epsilon^{2} G^{(2)}+\ldots \\
& F=1+\epsilon F^{(1)}+\epsilon^{2} F^{(2)}+\ldots \\
& \theta_{i}=p_{i} \zeta+q_{i} \tau+\phi_{i}, \quad i=1,2
\end{aligned}
$$

and require that the series truncate.

The Hirota method is well adapted to the case when Eq. (11) reduces to a bilinear equation or can be split into two or more independent (and consistent) bilinear equations. Unfortunately, further splitting of Eq. (11) into two bilinear equations is impossible in the general case (the direct naïve splitting of Eq. (11) into two equations corresponding to the two expressions in square brackets [13,7] is incorrect, since it gives $p_{1}=p_{2}$ and $q_{1}=q_{2}$ for the 2-soliton substitution (12) which is forbidden). Direct analysis of the multilinear equation (11) is not successful either. We shall try further reduction by substituting 


$$
G=\bar{G} \exp [i(\kappa \zeta-\Omega \tau)]
$$

into Eqs. (10) and (11). Choosing $\kappa=\Omega^{2}-\Omega^{3}$ we retain the structure of Eq. (10), while Eq (11) takes the following form:

$$
\begin{aligned}
& (\bar{G} \cdot F)\left[-i(1-3 \Omega) D_{\tau}^{2}(F \cdot F)+i\left(1-\Omega \gamma_{1}\right)\left(\bar{G}^{*} \cdot \bar{G}\right)+\left(\gamma_{1}+\gamma_{2}\right) D_{\tau}\left(\bar{G}^{*} \cdot \bar{G}\right)\right] \\
& +D_{\tau}(\bar{G} \cdot F)\left[-3 D_{\tau}^{2}(F \cdot F)+\left(3 \gamma_{1}+2 \gamma_{2}\right)\left(\bar{G}^{*} \cdot \bar{G}\right)\right]=0 .
\end{aligned}
$$

Selecting $\Omega=1 / 3$ or $\Omega=1 / \gamma_{1}$ eliminates the terms $\propto D_{\tau}^{2}(F \cdot F)$ or $\left(\bar{G}^{*} \cdot \bar{G}\right)$ in the first part of Eq. (16), respectively. Further simplification can be achieved in the particular case $\gamma_{1}=3$ when the choice $\Omega=1 / 3$ leads to the following equation

$$
D_{\tau}(\bar{G} \cdot F)\left[-3 D_{\tau}^{2}(F \cdot F)+\left(9+2 \gamma_{2}\right)\left(\bar{G}^{*} \cdot \bar{G}\right)\right]+(\bar{G} \cdot F)\left(3+\gamma_{2}\right) D_{\tau}\left(\bar{G}^{*} \cdot \bar{G}\right)=0 .
$$

Note that the first Hirota equation (10) implies that any $N$-soliton solution which takes the Hirota form can be written as $G / F=\Lambda\left(\gamma_{2}\right) \mathcal{G} / F$, where $\mathcal{G}$ and $F$ are independent of the parameter $\gamma_{2}$ (cf. [11,13,7]). Substituting this into (17) shows that this is possible only if $\gamma_{2}=-3$ or $\ln \left(\mathcal{G}^{*} / \mathcal{G}\right)=$ const. The first choice corresponds to the case described in Ref. [11]. We shall consider here the second choice which leaves $\gamma_{2}$ arbitrary.

Returning to Eqs. (3) we substitute

$$
A=\mathcal{E}(\zeta, \tau) \exp [i(\kappa \zeta-\Omega \tau)]
$$

which gives:

$$
\begin{aligned}
& \mathcal{E}_{\zeta}=-i\left(\kappa+\Omega^{2}-\Omega^{3}\right) \mathcal{E}+\left(2 \Omega-3 \Omega^{2}\right) \mathcal{E}_{\tau}+i(1-3 \Omega) \mathcal{E}_{\tau \tau} \\
& +i\left(1-\Omega \gamma_{1}\right)|\mathcal{E}|^{2} \mathcal{E}+\mathcal{E}_{\tau \tau \tau}+\gamma_{1}\left(|\mathcal{E}|^{2} \mathcal{E}\right)_{\tau}+\gamma_{2} \mathcal{E}\left(|\mathcal{E}|^{2}\right)_{\tau}
\end{aligned}
$$

Choosing $\kappa=\Omega^{3}-\Omega^{2}$ and making the coordinate transformation $T=\tau+\left(2 \Omega-3 \Omega^{2}\right) \zeta$, eliminates the terms $\propto \mathcal{E}$ and $\mathcal{E}_{\tau}$. Selecting $\Omega=1 / 3$ or $\Omega=1 / \gamma_{1}$, eliminates terms $\propto \mathcal{E}_{\tau \tau}$ or $|\mathcal{E}|^{2} \mathcal{E}$, respectively. In the particular case $\gamma_{1}=3$, $\Omega=1 / 3$, one arrives at the following complex modified $\mathrm{KdV}$ equation:

$$
\mathcal{E}_{\zeta}=\mathcal{E}_{T T T}+3\left(|\mathcal{E}|^{2} \mathcal{E}\right)_{T}+\gamma_{2} \mathcal{E}\left(|\mathcal{E}|^{2}\right)_{T}
$$

which is more general than the equation considered in Ref. [14] since it is for arbitrary $\gamma_{2}$. It is easy to see that, for the special case $\gamma_{1}=3$, Eq. (3) has an $N$-soliton solution of the form:

$$
A=B(\zeta, T) \exp [i(-2 \zeta / 27-\tau / 3+\psi)], \quad T=\tau+\zeta / 3, \quad \psi=\text { const }
$$

where $B(\zeta, T)$ is the $N$-soliton solution of the real modified $\mathrm{KdV}$ equation

$$
B_{\zeta}=B_{T T T}+\left(9+2 \gamma_{2}\right) B^{2} B_{T},
$$

and can be written as [5]

$$
\begin{aligned}
& B=i\left(\frac{6}{9+2 \gamma_{2}}\right)^{1 / 2}\left(\ln \left(f^{*} / f\right)\right)_{T}, \\
& f=\sum_{\mu=0,1} \exp \left(\sum_{i=1}^{N} \mu_{i}\left(\eta_{i}+i \pi / 2\right)+\sum_{1 \leq i<j}^{N} \mu_{i} \mu_{j} A_{i j}\right), \\
& \eta_{i}=q_{i} T+q_{i}^{3} \zeta+\eta_{i}^{(0)}, \quad A_{i j}=\ln \left(\frac{q_{i}-q_{j}}{q_{i}+q_{j}}\right)^{2} .
\end{aligned}
$$

This $N$-soliton solution differs from that proposed in Ref. [7] (which does not fulfill the corresponding nonlinear equation). Note that the existence of $N$-soliton solutions does not in general imply integrability. However, the integrability of the complex modified $\mathrm{KdV}$ in the special case of $\gamma_{1}=3$ and $\gamma_{2}=-3 / 2$ has been demonstrated in Ref. [14].

Finally, we study the integrability of the HONSE, applying the Painleve analysis [8 10]. It is widely believed that possession of the Painlevé feature is a sufficient criterion for integrability (see discussion in Refs. [15, 16]). The PDE in Eq. (3) can be analyzed to ascertain whether it is integrable by seeking a solution of the PDE in the Painlevé 
form $A(\zeta, \tau)=(a(\zeta, \tau))^{-\sigma} \sum_{m=0}^{\infty} b_{m}(\zeta)(a(\zeta, \tau))^{m}$, where $b_{m}(\zeta)$ are analytic functions of $\zeta$ in the neighborhood of a noncharacteristic movable singularity manifold defined by $a(\zeta, \tau)=\tau-f(\zeta)$. Following Ref. [16] we substitute into Eq. (3) the following Laurent series

$$
\begin{aligned}
& A=\left(\tau-\tau_{0}\right)^{-\sigma} \sum_{m=0} R_{m}(\zeta)\left(\tau-\tau_{0}\right)^{m}, \\
& A^{*}=\left(\tau-\tau_{0}\right)^{-\sigma} \sum_{m=0} S_{m}(\zeta)\left(\tau-\tau_{0}\right)^{m},
\end{aligned}
$$

(in the vicinity of a movable singular point $\tau=\tau_{0}(\zeta)$. Inspection of the strongest singularity immediately gives $\sigma=1$ and $R_{0} S_{0}=-6 /\left(3 \gamma_{1}+2 \gamma_{2}\right)$. The subsequent substitution of (26) and (27) into Eq. (3) should allow identification of the other $R_{m}$ and $S_{m}$ leaving exactly 6 arbitrary functions (including $\tau_{0}(z)$ ) undetermined. Analysis of resonances is carried out by making an auxiliary substitution of the form $A=R_{0}\left(\tau-\tau_{0}\right)^{-1}+R_{p}\left(\tau-\tau_{0}\right)^{p-1}$ (and similarly for $A^{*}$ ) into the PDE and retaining only linear terms in $R_{p}$ (cf. Ref. [5, [7]). This procedure shows that resonances occur at $p=-1,0,3,4$, and $3 \pm 2 \sqrt{-\gamma_{1} /\left(3 \gamma_{1}+2 \gamma_{2}\right)}$. The resonances at $p=-1$ and $p=0$ correspond to the arbitrariness of $\tau_{0}$ and $S_{0} / R_{0}$, respectively. Requiring that the resonant indices $p$ be integers [16] one finds that either $\gamma_{1}=-2 \gamma_{2}$ or $\gamma_{1}=-\gamma_{2}$. Further substitution of the complete power series of (26) and (27) into Eq. (3) shows that there are a sufficient number of arbitrary functions if and only if $\gamma_{1}=3$ and $\gamma_{2}=-3 / 2$. This corresponds to the case studied in Ref. 14]. Thus, the parameters that insure the Painlevé feature are a proper subset of the parameters that insure existence of an $N$-soliton solution of the HONSE.

To summarize, we have derived a 1-soliton solution of the general HONSE, without any constraints on its coefficients except for the weak inequality constraint $3 \gamma_{1}+2 \gamma_{2}>0$. The 1-soliton width and intensity are related by the expression $I_{s} \tau_{s}^{2}=6 /\left(3 \gamma_{1}+2 \gamma_{2}\right)$, so the intensity decreases with increasing $\left(3 \gamma_{1}+2 \gamma_{2}\right)$ for a given $\tau_{s}$. We have shown that $N$-soliton solutions exist when $\gamma_{1}=3$ and $\gamma_{2}$ is arbitrary, thus extending significantly the results of Ref. [11,14. The Painlevé plausibility condition for integrability, $\gamma_{1}=-2 \gamma_{2}=3$, has been shown to be a proper subset of the conditions for $N$-soliton solutions, and consistent with the integrable case found in Ref. [14, while integrability of our more general case is still unknown. Our analysis does not exclude existence of $N$-soliton solutions nor does it disprove integrability for arbitrary $\gamma_{1}$ and $\gamma_{2}$.

\section{ACKNOWLEDGMENTS}

This work was supported in part by a grant from the US-Israel Binational Science Foundation.

[1] Y. Kodama and A. Hasegawa, IEEE J. Quantum Electron. QE-23, 5610 (1987).

[2] F. M. Mitschke and L. F. Mollenauer, Opt. Lett. 11, 657 (1986).

[3] A. Hasegawa, Optical Solitons in Fibers, (Springer, Heidelberg, 1989).

[4] G. P. Agrawal, Nonlinear Fiber Optics, (Academic Press, NY, 1989).

[5] M.A. Ablowitz and H. Segur, Solitons and the inverse scattering transform, (SIAM, Philadelphia, 1981).

[6] A. Hasegawa and F. Tappert, Appl. Phys. Lett. 23, 142 (1973).

[7] K. Porsezian and K. Nakkeeran, Phys. Rev. Lett. 76, 3955 (1996).

[8] J. Weiss, M. Tabor, and G. Carnevale, J. Math. Phys. (NY) 24, 522 (1983).

[9] M. Musette and R. Conte, J. Math. Phys. (NY) 32, 1450 (1991).

[10] M. J. Ablowitz and P.A. Clarkson, Solitons, Nonlinear Evolution Equations and Inverse Scattering, (Cambridge University Press, Cambridge, 1991).

[11] R. Hirota, Phys. Rev. Lett. 27, 1192 (1971); J. Math. Phys. (NY) 14, 805 (1973).

[12] The Padé approximant in theoretical physics, Eds. G.A. Baker and J.L. Gammel, (Academic Press, NY, 1970).

[13] Shan-liang Liu and Wen-zheng Wang, Phys. Rev. E 49, 5726 (1994).

[14] N. Sasa and J. Satsuma, J. Phys. Soc. Jpn. 60, 409 (1991).

[15] A.C. Newell, Solitons in mathematics and physics, (SIAM, Philadelphia, 1985).

[16] A. Ramani, B. Grammaticos, and T. Bountis, Phys. Rep. 180, 159 (1989). 